Infusion Therapy

Transfusion Medicine

Infusionstherapie

Transfusionsmedizin
Meetings and Conferences - Tagungen und Kongresse

Infus Ther Transfus Med 2000;27:324

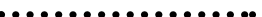

07.02.-09.02.2001

Bremen

Deutschland
11. Internationales Symposium Intensivmedizin/Intensivpflege

45. Jahrestagung der Gesellschaft für Thrombose- und Hämostaseforschung

Düsseldorf

Deutschland

\subsection{3.-17.03.2001}

Bremen

Deutschland

\section{Bremer Palliativkongress}

\subsection{3.-30.03.2001}

Regensburg

Deutschland
Cellular Therapy 2001

6th Meeting of the European Haematology Association

21.06.-24.06.2001

Frankfurt/M

Deutschland

\subsection{6.-23.06.2001}

Duisburg

Deutschland

[...................

Riva del Garda

Italy

\subsection{9.-26.09.2001}

Lubljana

Slovenia

\subsection{9.-28.09.2001 \\ Hamburg}

Deutschland

\subsection{8.-28.08.2002}

Vancouver

Canada

Cellular Therapy 2001

Update Hämatologie/Onkologie 2001 Immunhämatologie e.V. 13th Congress of the European Society for Haemapheresis

2nd International Postgraduate School of Immunogenetics in Transfusion Medicine: Molecular and Cellular Basis of Immunity and Alloimmunity

34. Kongress der Deutschen Gesellschaft für Transfusionsmedizin und

27th Congress of the International Society of Blood Tranfusion
Auskunft: Prof. Dr. W. Kuckelt, Zentrum für Anästhesiologie, Klinik für operative und allgemeine Intensivmedizin, Zentralkrankenhaus Links der Weser, Senator-Weßling-Straße 1, D-28277 Bremen Tel. +49 421 87-917 31, Fax -917 88 oder 29988 E-mail Kuckelt@intensivmed.de www.intensivmed.de

Auskunft: Porstmann Kongresse GmbH, Friedrichstraße 130a, D-10117 Berlin

Tel. +49 302844 99-0, Fax -11

E-mail porstmann@porstmann-kongresse.de www.gth-online.de

Auskunft: Dr. med. H.-J. Willenbrink, Abteilung für Schmerzdiagnostik, Schmerztherapie und Palliativmedizin, Zentrum für Anästhesiologie, Zentralkrankenhaus Links der Weser, Senator-Weßling-Straße 1, D-28277 Bremen

Tel. +49 421 879-17 86, Fax -14 63

www.zkhldw.de/palliativ.htm

Auskunft: Gertraud Geißler, Abteilung Hämatologie/ Onkologie, Universität Regensburg, Franz-JosefStrauß-Allee 11, D-93053 Regensburg Tel. +49 941944 55-01, Fax -02

E-mail gertraud.geissler@klinik.uni-regenburg.de www.cellular-therapy2001.de

Auskunft: Eurocongres Conference Management, Jan van Goyenkade 11, NL-1075 HP Amsterdam Tel. +31 20 67-934 11, Fax -373 06

E-mail eha2001@eurocongres.com www.eurocongres.com/eha2001

•.......................

Auskunft: Prof. Dr. S. Petrasch, Klinikum Duisburg, Zu den Rehwiesen 9, D-47055 Duisburg Tel. +49 203733 23-00, Fax -02

.............................

Auskunft: S.I.d.E.S. Società Italiana di Emaferesi Servizi, Via Paolo Emilio 7, I-00192 Roma Tel. +3906 32 35-51 21, Fax -56 48

E-mail infoside@mix.it

www.mix.it/side

Auskunft: Resear Centre for Transfusion Medicine of the Republic Slovenia, Šlajmerjeva 6, SL-1000 Lubljana

Tel. +386 61-543 8147 oder 54381 00, Fax -30 2224 E-mail mateja.bohinjec@mf.uni-lj.si http://schimmgen.zrs-tk.si

Auskunft: Kongress- und MesseBüro Lentzsch GmbH, Seifgrundstraße 2, D-61348 Bad Homburg Tel. +49 617267 96-0, Fax -26 E-mail Lentzsch@aol.com www.dgti.de

-..........................

Auskunft: SBT 2002 Secretariat, c/o Venue West Conference, Services Ltd., 645 - The Landing, 375 Water Street, Vancouver, BC, Canada V6B 5C6 Tel. +1 604 681-52 26, Fax -2503 www.isbt2002.com 